\title{
A modified agile-eye mechanism for robotic manipulation of wristed laparoscopic instruments
}

\author{
Alireza Alamdar ${ }^{1,2}$, Shahrzad Hanifeh ${ }^{3}$, Farzam Farahmand ${ }^{1}$, Alireza Mirbagheri ${ }^{3,4}$
}

\begin{abstract}
Replacement of the exclusively designed instruments of the robotic surgery systems with the commercial hand-held wristed instruments provides advantages such as single-usability and cost reduction. A 4-DOF robotic system, based on a modified non-symmetric 2-DOF agile-eye mechanism, was developed to manipulate the hand-held wristed instruments. The kinematics of the mechanism was analyzed, its dimensions were optimized, and a functional prototype was tested experimentally. The optimized mechanism had a great kinematic isotropy (condition number $<1.31)$ in the target workspace. Experimental studies revealed a high tracking accuracy $\left(0.27^{\circ} \pm 0.01^{\circ}\right.$ rms for the worse case) and a reasonably acceptable compliance $(0.19 \mathrm{deg} / \mathrm{N} . \mathrm{m}$ and $0.45 \mathrm{deg} / \mathrm{N} . \mathrm{m}$ for the first and second kinematic chains respectively). By satisfying the design requirement, the robotic manipulator provides an attractive choice for robotic surgery systems. The performance of the manipulator can be improved further by increasing the stiffness of the second kinematic chain and performing kinematic calibration.

Keywords: Robotic surgery, Wristed laparoscopic instruments, 2-DOF agile-eye mechanism, Design optimization
\end{abstract}

\section{INTRODUCTION}

The currently available robotic surgery systems, e.g., Da Vinci surgical system (Intuitive Surgical Inc.), employ exclusively designed wristed laparoscopic instruments for performing minimally invasive robotic surgery (MIRS) procedures. These instruments, e.g., EndoWrist (Intuitive Surgical Inc.), have been designed to provide surgeons with a great level of dexterity. However, they are expensive and often used as reusable devices (for up to 10 times) [1], [2].

An alternative solution to make the surgical instruments less costly and single-use, is to enable the surgical robot to work with commercial hand-held wristed laparoscopic instruments [3], [4]. There are several models of such instruments available in the market and used in clinical practice, e.g., RealHand (Novare Surgical Systems, Inc.) [5], Autonomy Laparo-Angle Articulating Instruments (Cambridge Endoscopic Devices, Inc.) [6], and SILS Hand Instruments (Medtronic-Covidien) [7], [8], [9]. It has been reported that these instruments have not well received in their primary target application, i.e., conventional (non-robotic) minimally invasive surgery, due to requiring unergonomic handle maneuvers and applying excessive forces to the incision point [10], [11], [12]. However, their integration

\footnotetext{
1 Authors are with Department of Mechanical Engineering, Sharif University of Technology, Tehran, Iran. Corresponding author: Alireza Alamdar (aalamda3@jhu.edu).

${ }^{2}$ Author is also with Laboratory for Computational Sensing and Robotics, Johns Hopkins University, Baltimore, MD, USA.

3 Authors are with Research Center for Biomedical Technologies and Robotics (RCBTR), Advanced Medical Technologies and Equipment Institute (AMTEI), Tehran University of Medical Sciences, Tehran, Iran.

${ }^{4}$ Author is also with Medical Physics and Biomedical Engineering Department, School of Medicine, Tehran University of Medical Sciences, Tehran, Iran.
}

into the surgical robotic system is promising, considering their low prices and the fact that the reported problems are resolved when the instruments are manipulated robotically.

For robotic manipulation of the hand-held wristed instruments, there is a need to a 4-DOF robotic manipulator. Two of these DOFs involve controlling the roll and grasping motions of the instrument which might be performed using a simple 1-DOF actuator unit each. The other two DOFs are associated with the manipulation of the snake-like distal wrist of the instrument, for which a remote center of motion (RCM) mechanism is required to move the handle of the instrument around a spherical joint at the interface of the handle and the shaft (Fig. 1).

A vast variety of 2-DOF RCM mechanisms have been proposed in the literature to be used in MIRS systems, among which, the parallelograms, the spherical mechanisms, and the circular tracking arcs are most prevalent [13]. The parallel RCM mechanisms, e.g., spherical parallel mechanism (SPM), gimbal mechanism, and parallel wrist mechanism, have been also suggested for MIRS application considering their high rigidity to size ratio and low moving inertia [14]. Among different variations of parallel RCM mechanisms, SPMs are most attractive for their compact structure, great manipulability and low moving inertia [15], [16], [17] and have been proposed for various surgical applications such as camera holding [18], bone flap removal [19], laparoscopic surgery [3], [20], teleechography [21] and others [22].

The previously reported applications of 2-DOF SPMs in the literature, have been all based on the orthogonal, i.e., agileeye [23], and symmetric designs of the mechanism. Although agile-eye mechanism provides great isotropy and covers the whole $S^{2}$ workspace, its large links can be problematic when the size and weight are important and a smaller workspace is sufficient. Moreover, for MIRS applications, smaller links can help to resolve the internal and external collision problem and improve the precision by increasing the stiffness of the structure.

This study reports the design, development and validation of a 4-DOF robotic system for manipulation of hand-held wristed laparoscopic instruments. The proposed robotic manipulator consists of a modified non-symmetric agile-eye mechanism for manipulation of the distal wrist of the instrument, as well as actuation units for its roll and grasp DOFs. In the following, first, the design considerations of the robotic manipulator are described, based on the geometrical, kinematic, and force characteristics of the instrument, to design the robot conceptually. Then the kinematics of the modified agile-eye mechanism is analyzed in detail, and its dimensions are optimized. Finally, a functional prototype of the robotic manipulator is fabricated and its performance in meeting the design considerations is 
assessed experimentally.

\section{Materials AND MethodS}

\section{A. Conceptual Design}

A wide range of commercial hand-held wristed laparoscopic instruments use a snake-like distal wrist, which is mechanically actuated by a handle pivoted about the instrument's rod [10], [12]. Among them, RealHand (Novare Surgical Systems, Inc.) [5], Autonomy Laparo-Angle Articulating Instruments (Cambridge Endoscopic Devices, Inc.) [6], and SILS Hand Instruments (Medtronic-Covidien) [7] are considered the best in meeting the surgeon's requirements [10]. These instruments are $5 \mathrm{~mm}$ in diameter, single use, very affordable and provide the surgeons with 2-DOF wrist motion, distal roll, and grasp maneuver [10], [12]. They also have the same method of actuation: the distal wrist is oriented by rotating the handle about the spherical joint, connecting the handle to the shaft (see Fig. 1(a)); the continuous distal roll is provided by the knob on the handle; and the grasp is provided by moving the handle grip. Therefore, a robotic manipulator with modular design might be able to integrate all these instruments to the host surgical robot.

The hand-held wristed laparoscopic instrument used in this study was a Medtronic-Covidien SILS Hand Instrument (Fig. 1). In order for the robotic manipulator to receive the instrument properly, its handle was modified slightly. The modification included adding an interface for actuation of the roll/grasp and a cautery connector for execution of the electrocautery. Then the workspace and force requirements of manipulation of the instrument wrist though its handle were investigated.

It was found that for complete bending (up to $75^{\circ}$ ) and distal roll ( $360^{\circ}$ continuous) of the instrument tip, there is need to $\pm 23^{\circ}$ rotation of the handle around the spherical joint at the interface of the handle and the shaft of the instrument. Also the required torque for complete bending, while holding a $500 \mathrm{gr}$ weight by the tool-tip, was measured to be $1.3 \mathrm{~N} . \mathrm{m}$. Moreover, the required displacement and force for grasp motion of the instrument, for firmly holding a needle, were found as $5 \mathrm{~mm}$ and $100 \mathrm{~N}$, respectively, and the required torque for continuous roll as 0.5 N.m.

The mechanical and functional characteristics of the wristed laparascopic instrument were used to capture the design requirements of the robotic manipulator. For the degrees of freedom, the manipulator should be able to support four DOFs; two for the wrist motion and one for each of the roll and the grasp. The wrist motion shall be controlled via an RCM which holds the instrument shaft and moves the handle around the spherical joint. The roll and grasp motions, on the other hand, might be controlled using independent actuation units fixed to the end-effector of the RCM mechanism to move and rotate the roll/grasp interface with respect to the handle. For effective manipulation of the instrument, the design shall satisfy the following requirements:

- Collision-free workspace: The RCM mechanism should be able to maneuver the handle across its required workspace, i.e. a cone with apex angle of $50^{\circ}$, and also

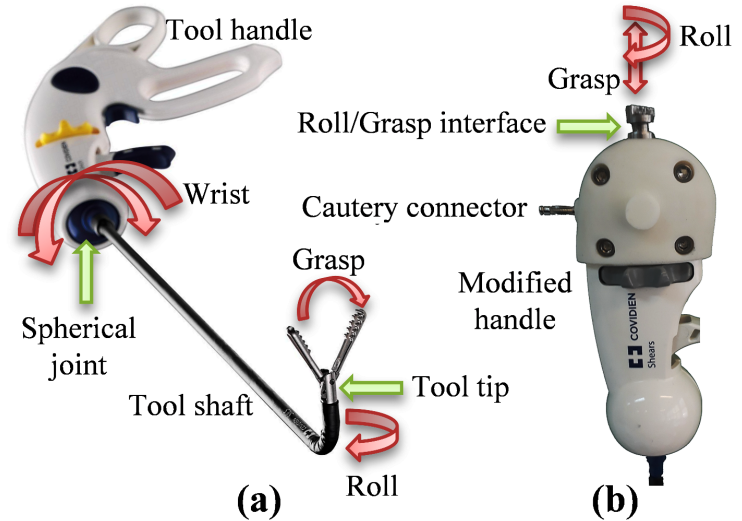

Fig. 1. (a) Original Medtronic-Covidian SILS instrument and (b) modified handle for robotic actuation

avoid collision with tool shaft, host robotic arm, and its own links. Also, the roll/grasp actuation units should provide a cylindrical motion, for actuating the roll/grasp interface, consisting of a continuous roll and $5 \mathrm{~mm}$ range of motion for the grasp.

- Minimum size and inertia: Considering the fact that the robotic manipulator is attached to the distal end of the host robotic arm, the weight and size of its structure, including the base unit and the moving parts, should be minimized as much as possible.

- High tracking accuracy: The robotic manipulator should provide a sufficiently high tracking accuracy in performing the surgical maneuvers.

- Modular and interchangeable design: During operation, the surgeon may need to repeatedly switch between different instruments, e.g. grasper, scissor, etc. So the designed manipulator should provide the means for easy and fast attachment/detachment of the instrument. Moreover, all parts of the manipulator that are in contact with the instrument should be sterilizable.

Considering the above requirements, SPMs seem a preferred choice for their compact structure, large workspace and great dynamic performance, as well as high rigidity and low moving inertia [15], [16], [17], due to the closed kinematic chain and stationary motors. A simple orthogonal 5R-SPM, i.e. Agile Eye [23], however, has been shown to be incapable of meeting the collision free design requirement of the manipulator [3]. Hence, this study investigated the performance of a new nonsymmetric structure of 5R-SPM, with orthogonal links in one chain and links with arbitrary lengths in the other [24], for this application (Fig. 2). The smaller links in the second kinematic chain can help avoiding collisions, reducing the size of the mechanism, and increasing its stiffness.

The schematics of the proposed non-symmetric agile-eye mechanism is shown in Fig. 2. It consists of four moving links and five revolute joints. The joints are denoted by $\theta_{i}$, $i=1,2,3,4,5$, with the joint axes along $\mathbf{Y}_{0}, \mathbf{X}_{2}, \mathbf{Z}_{3}, \mathbf{Y}_{E E}$ and $\mathbf{X}_{1}$, respectively. The axes of all joints intersect at a common point, i.e. the remote center of motion (RCM). The joints 1 and 2 are the active joints and indicate the orientation of the 


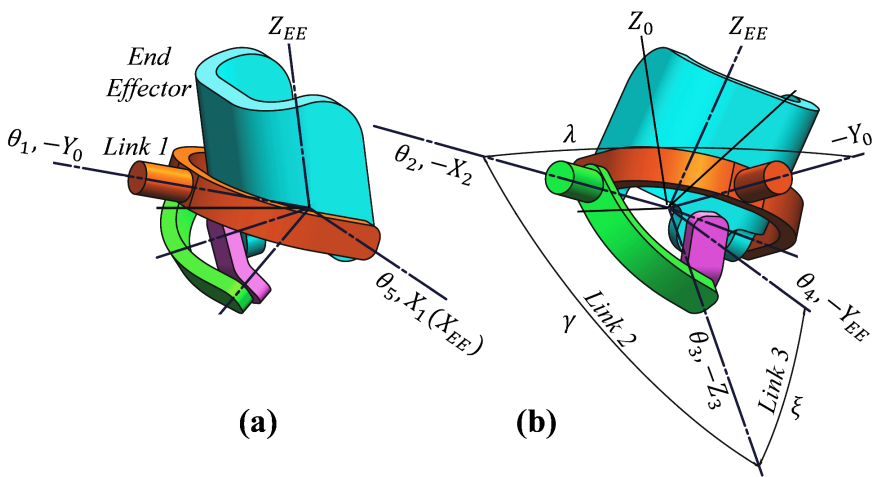

Fig. 2. Coordinate assignments and link and joint parameters for the proposed non-symmetric agile-eye in (a) side and (b) rear views. $X_{0} Y_{0} Z_{0}$ denotes the agile-eye's reference coordinate system, $\theta_{i}, i=1,2,3,4,5$ denote the joints along $\mathbf{Y}_{0}, \mathbf{X}_{2}, \mathbf{Z}_{3}, \mathbf{Y}_{E E}$ and $\mathbf{X}_{1}$ axes, $\lambda$ denotes the angle between the two active joints $\theta_{1}$ and $\theta_{2}$, and $\gamma$ and $\xi$ denote the length of links 2 and 3 , respectively.

end-effector, $\mathbf{Z}_{E E}$.

The mechanism has two kinematic chains. The first chain is orthogonal, i.e $\mathbf{Y}_{0} \perp \mathbf{X}_{1}$ and $\mathbf{X}_{1} \perp \mathbf{Z}_{E E}$ (Fig. 2(a)). In the commonly used agile-eye mechanism, the second chain is also orthogonal. However, here we used a modified design, in which the link lengths of the second chain are less than $\pi / 2$. The dimensions of this chain are characterized by $\lambda$, denoting the angle between the two active joints, and $\gamma$ and $\xi$, indicating the lengths of links 2 and 3, respectively.

\section{B. Kinematic Optimization}

A thorough kinematic and singularity analysis of the proposed non-symmetric agile-eye mechanism is provided in [24]. According to the results, the mechanism has four working modes, i.e. four solutions for the inverse kinematics, and two assembly modes, i.e. two solutions for the forward kinematics. The inverse singularities occur in $\cos \theta_{3}=0$ or $\cos \theta_{5}=0$ and the forward singularity occurs in $\cos \theta_{4}=0$.

In order for the manipulator to never pass through a singularity, it should be optimized such that the required workspace remains inside a single assembly mode and a single working mode. A preliminary analysis on the CAD model showed that the assembly mode with $\cos \theta_{4}>0$ and the working mode with $\cos \theta_{3}>0$ and $\cos \theta_{5}>0$ results in less collision problems between the links of the mechanism and the instrument shaft. Then, a kinematic optimization was performed to best fulfill the design considerations mentioned in section II-A.

1) Optimization parameters: Five optimization parameters were considered for the mechanism, two of which in relation to the placement of mechanism on the robot base ( $\alpha$ and $\beta$ in Fig. 3) and three of which corresponding to the mechanism link lengths ( $\lambda, \gamma$, and $\xi$ in Fig. 2).

The range of $\alpha$ and $\beta$ were considered as $0^{\circ} \leq \alpha \leq \lambda / 2$ and $-10^{\circ} \leq \beta \leq 10^{\circ}$. Exceeding these limits would gradually increase the size of the mechanism and also make the design more complicated. The angle between two active joints, $\lambda$, was set between $50^{\circ}$ and $100^{\circ}$, lower values being technically unfeasible, due to the size of the motors, and larger values

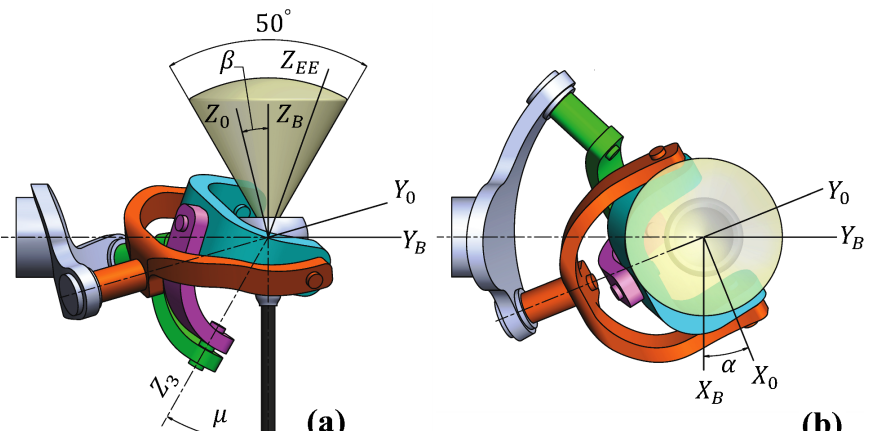

(b)

Fig. 3. Coordinate assignments and base placement parameters for the proposed non-symmetric agile-eye in (a) side and (b) top views. $X_{B} Y_{B} Z_{B}$ denotes the robot's base frame, and $X_{0} Y_{0} Z_{0}$ denotes the agile-eye's reference coordinate system, which is transformed from $X_{B} Y_{B} Z_{B}$ by means of two rotations $\alpha$ and $\beta$.

adding too much to the size of the mechanism. Finally, the link lengths $\gamma$ and $\xi$ were ranged between $15^{\circ}$ and $90^{\circ}$. For larger values of $\gamma$ and $\xi$, avoiding collision between the links and the instrument shaft becomes almost impossible.

After establishing the range of the optimization parameters, the trial optimization sets were generated using nested loops by applying $1^{\circ}$ increments to the optimization parameters. Then, for each optimization set, the target workspace was discretized into a number of search points, and for each search point, the optimization criteria were assessed.

2) Optimization criteria: The design considerations, mentioned in section II-A, were formulated into quantitative measures to establish the optimization constraints and cost function.

From workspace point of view, an optimization set is valid if it covers the whole workspace without any collision between the links and the instrument shaft. The proposed mechanism is expected to maneuver the handle inside a cone with vertical axis (in $\mathbf{Z}_{B}$ direction) and an apex angle of $50^{\circ}$ (Fig. 3). For each search point, the orientation of the tool handle (mechanism end-effector) with respect to agile-eye's reference coordinate system, $X_{0} Y_{0} Z_{0}$, is:

$$
{ }^{0} \mathbf{Z}_{E E}=\left[\boldsymbol{R}_{Z}(\alpha) \boldsymbol{R}_{X}(\beta)\right]^{T} \boldsymbol{R}_{Z}(\theta) \boldsymbol{R}_{Y}(\phi)\left[\begin{array}{lll}
0 & 0 & 1
\end{array}\right]^{T}
$$

where $\theta$ and $\phi$ are the spherical coordinates of $\mathbf{Z}_{E E}$ with respect to robot's base frame, $X_{B} Y_{B} Z_{B}$, in Fig. 3 and range between $0^{\circ}$ to $360^{\circ}$ and $0^{\circ}$ to $25^{\circ}$, respectively. In order to ensure a collision-free workspace, after solving inverse kinematics for each search point, the angle between axis of the third joint and orientation of the instrument shaft ( $\mu$ in Fig. 3) was calculated as

$$
\begin{gathered}
\mu=\arccos \left[\begin{array}{lll}
\sin \beta( & \cos \lambda & \left.\cos \gamma-\sin \lambda \sin \gamma \sin \theta_{2}\right) \\
+\cos \beta & \sin \lambda & \cos \theta_{2}
\end{array}\right]
\end{gathered}
$$

An optimization set was discarded, if, even for one search point, $\mu$ became less than $10^{\circ}$.

To minimize the size of the motors and also maximize their performance in transmitting the force and motion to the endeffector, the kinematic isotropy index was employed. For each 
search point of each optimization set, the condition number, i.e., the ratio between the biggest and smallest eigenvalues of the Jacobian matrix, was calculated. The condition number ranges between one and infinity, one denoting completely isotropic and infinity meaning singular configuration. For each optimization set, the overall condition number, i.e. the average of the condition numbers for all search points, was used as a scoring measure in the cost function. Moreover, to keep the overall size of the mechanism minimum, $\alpha=\lambda / 2$ and $\beta=0^{\circ}$ are preferred. The former leads to symmetric positioning of the tool manipulator, when installed on the host robotic arm, and the latter results in less design complexity. Also, smaller angles for $\lambda$ yields a smaller size for the mechanism's base unit. Therefore, the terms $|\alpha-\lambda / 2|,|\beta|$, and $\lambda-50^{\circ}$ were also used as penalizing measures in the cost function.

To ensure a high tracking accuracy for the mechanism, two additional measures were included in the cost function. First, from kinematic point of view, increasing the stiffness of the mechanism enhances its tracking accuracy. Thus, smaller link lengths are preferred for the proposed non-symmetric agile-eye mechanism. Since the link stiffness is proportional to the inverse of the cube of the link length, the term $\gamma^{3}+\xi^{3}$ was considered as another penalizing measure in the cost function. Second, the tracking accuracy deceases near singular configurations. In order to account for this effect, an optimization set was discarded if, in even a single search point, it had a condition number greater than 2 or a determinant of Jacobain matrix lower that 0.2 . For the remaining optimization sets, maximum condition number was considered as a scoring measure in the cost function.

Finally, by normalizing the scoring and penalizing measures, the cost function was obtained as:

$$
\begin{aligned}
C F= & \frac{|2 \alpha-\lambda|+\lambda}{\lambda} \times \frac{|\beta|+10}{10} \times \frac{\lambda}{50} \\
& \times \frac{\gamma^{3}+\xi^{3}+2(\pi / 2)^{3}}{2(\pi / 2)^{3}} \times \max C N \times \overline{C N}
\end{aligned}
$$

where $C N$ denotes the condition number. Note that we used multiplication to dedicate equal weighting for each scoring and penalizing measure. Also, each term of the cost function is normalized in a way that it would range from 1 to 2 . This is necessary, since otherwise, closeness of one term to zero would diminish the effect of all the remaining terms.

\section{Experimental Examination}

A functional prototype of the optimized structure of robotic manipulator was fabricated for experimental examination (Fig. 4). The agile-eye links were precisely fabricated using 5 axis CNC machining. The materials used for fabricating the tool interface units were selected from bio-compatible materials such as aluminum, steel and PEEK. The cautery connection between the robot and the tool was secured via a handle holder unit. The main body of the handle holder and the shaft holder, as well as the part between the roll/grasp actuation interface and the tool were fabricated from PEEK material, to ensure insulation between the tool and the robot. We used Maxon EC Flat motors and zero backlash harmonic

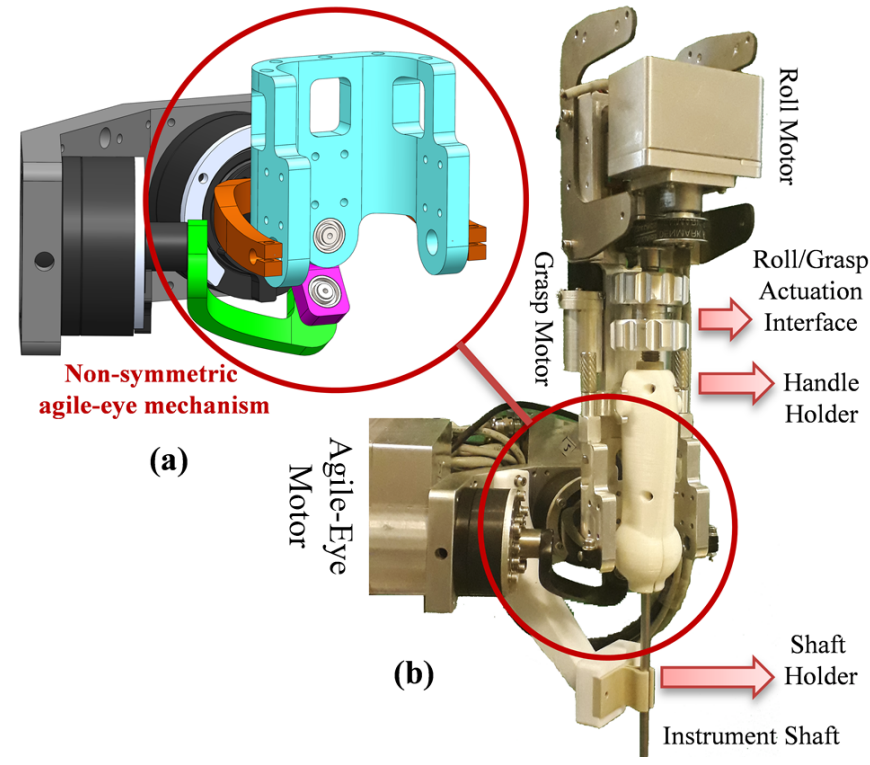

Fig. 4. (a) The CAD model, and (b) the functional prototype of the optimized structure of robotic manipulator. While the CAD model shows only the actuation units of the agile eye (the wrist joint motions), the functional prototype includes also the roll and grasp actuation units of the instrument, as well as the associated mechanical components, i.e., links, motor supports, tool interfaces, etc.

drive gearboxes for compact and precise actuation of the wrist and roll motions. For the grasp motion, we used an integrated Maxon spindle unit. MaxPos drivers were employed to command the motors, and TwinCAT software was used for real-time communication via EtherCAT protocol.

A series of tests were performed to assess the performance of the manipulator in fulfilling the design requirements. During the tests, the 3D position of the end-effector was captured by an infrared tracker (NDI Hybrid Polaris Spectra, Northern Digital Inc., Canada), with a capturing frequency of $60 \mathrm{~Hz}$ and an overall RMS tracking error of $250 \mu \mathrm{m}$. A set of two markers were assembled equally distanced from the RCM point via a connecting rod (see Fig. 5). By finding the 3D position of this marker set, both the RCM location and endeffector direction, denoted by $\mathbf{Z}_{A c t}$, were calculated. The captured data from the NDI tracker and the recorded data from the control software were synchronized by means of a synchronization port embedded in the camera control unit. The data recorded from the control software included the motors' encoder position and actual torque, denoted by $\boldsymbol{\theta}_{E n c}$ and $\boldsymbol{\tau}_{A c t}$, respectively.

Fig. 6 shows an overview of the performance assessment tests of the functional prototype. The first test series aimed to assess the kinematics, workspace, and tracking accuracy of the mechanism. The target workspace, i.e $\phi=0-25^{\circ}$ and $\theta=0-360^{\circ}$ in spherical coordinate system, was meshed into seven trajectories. In the first three trajectories, referred to as conical trajectories, the end-effector was commanded to sweep in $\theta$ direction with fixed values of $\phi=5^{\circ}, \phi=$ $15^{\circ}$, and $\phi=25^{\circ}$ respectively. The next four trajectories, referred to as in-plane trajectories, include sweeping a planar sector of the target workspace, with fixed values of $\theta=0^{\circ}$, 


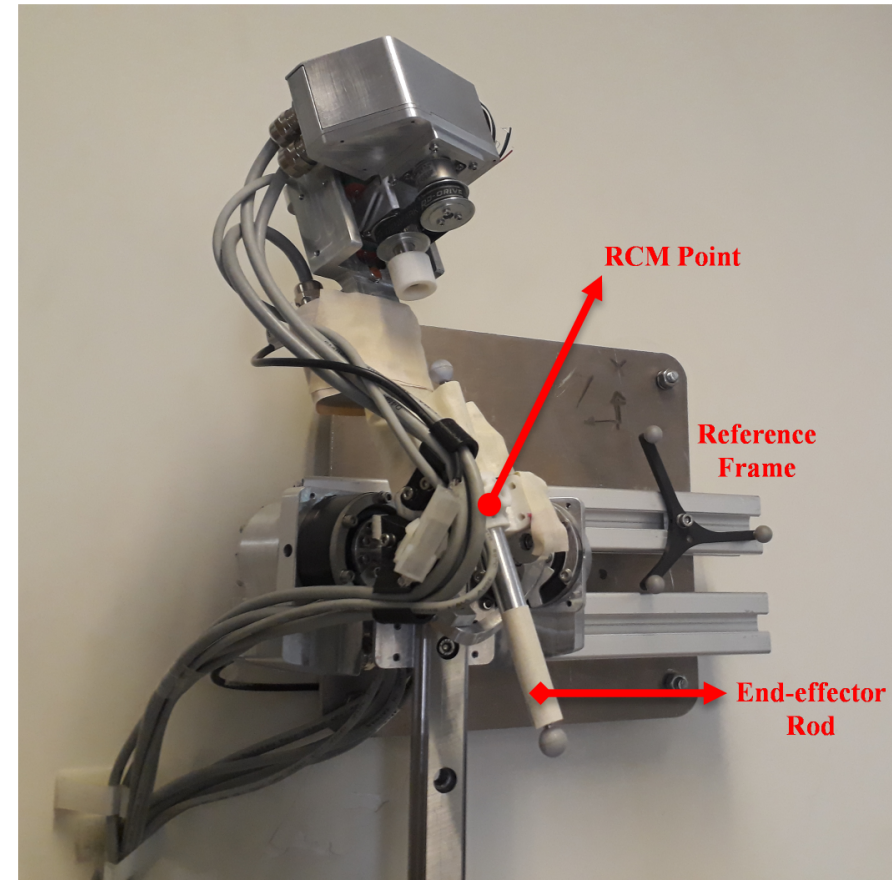

Fig. 5. Test setup including the base platform, tool manipulator and marker sets

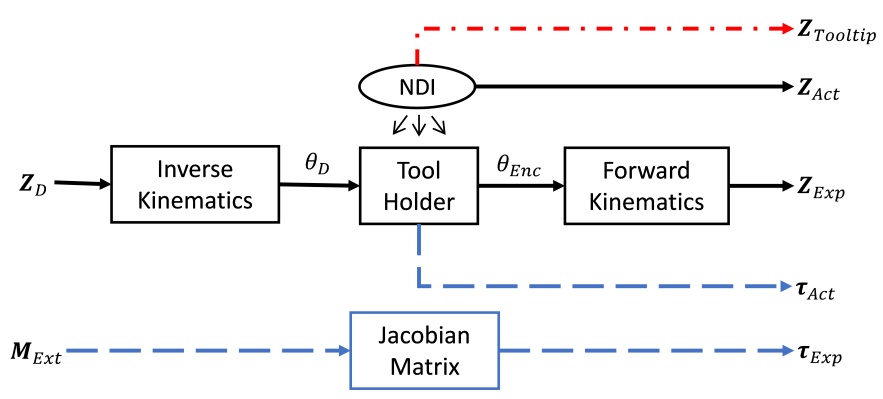

Fig. 6. Overview of performance assessment tests, including the kinematic tests, force response tests, and tool manipulation tests. $\mathbf{Z}_{D}, \mathbf{Z}_{E x p}$, and $\mathbf{Z}_{A c t}$ (or $\mathbf{Z}_{\text {Tooltip }}$ ) denote the desired trajectory, the expected trajectory (based on encoder's values), and the actual trajectory (measured by external tracker), respectively. Also $\mathbf{M}_{E x t}, \boldsymbol{\tau}_{A c t}$, and $\boldsymbol{\tau}_{E x p}$ stand for the external moment, the expected motor torques and the actual motor torques, respectively.

$\theta=45^{\circ}, \theta=90^{\circ}$, and $\theta=135^{\circ}$ and $\phi$ ranged from $-25^{\circ}$ to $25^{\circ}$. To evaluate the tracking accuracy, the expected and actual trajectories were compared and the angular difference was recorded. The expected trajectory, $\mathbf{Z}_{\text {Exp }}$, was calculated by solving the forward kinematics for $\boldsymbol{\theta}_{E n c}$, and the actual trajectory, $\mathbf{Z}_{\text {Act }}$, was captured by the camera (Fig. 6). To assess the repeatability of the mechanism, in following the defined trajectories, each test was repeated 10 times.

The next test series tended to examine the load carrying capability, stiffness and isotropy of the mechanism. As shown in Fig. 7(a), a constant load was applied to the agile-eye end-effector, by attaching constant force springs between the end-effector and a fixed overhead structure. The springs were selected such that they exert external moments equivalent to the required torques for manipulating the tool, as indicated in section II-A. The tests were performed for two conical trajectories, i.e. $\phi=15^{\circ}$ and $\phi=25^{\circ}$, and two springs,

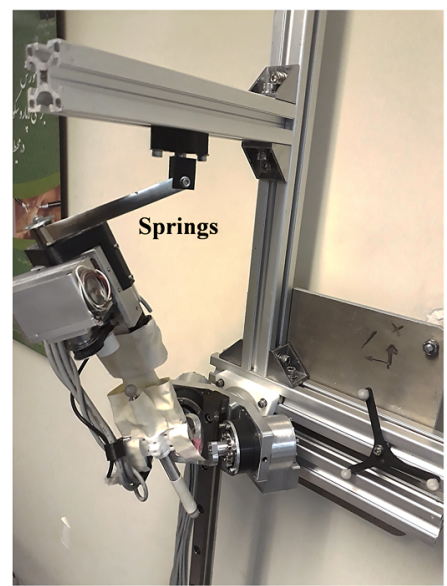

(a)

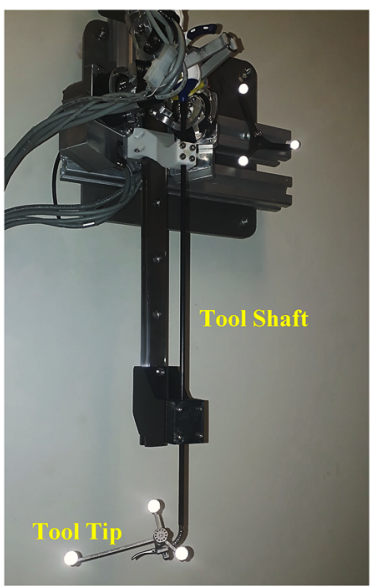

(b)
Fig. 7. Setup for (a) load-carrying capability, and (b) instrument's workspace coverage assessment tests.

$F_{s}=6.57 N$ and $F_{s}=11.67 N$, and similar outputs as the previous test sets were recorded.

Finally, the ability of the robot in manipulating the SILS instrument and providing required workspace for the wrist motion was evaluated. The instrument was attached to the tool manipulator and a marker set was attached to the tool-tip (see Fig. 7(b)). The three conical trajectories from first test series were commanded and the wrist angle, i.e. the orientation of the tool-tip with respect to the tool shaft, was recorded.

\section{RESULTS}

The optimization process suggested a non-symmetric design with link length parameters of $\lambda=64^{\circ}, \gamma=69^{\circ}$, and $\xi=35^{\circ}$ and base positioning parameters of $\alpha=32^{\circ}$ and $\beta=0^{\circ}$, as the optimum solution. Fig. 8 shows the condition number and the determinant of the Jacobian matrix for the optimum design, respectively. The condition number was lower than 1.31 and the determinant of the Jacobian ranged between 0.7 to 1.71 in the target workspace. Table 1 compares the structure and the performance measures of the non-symmetric agile-eye mechanism designed in this study with those of the orthogonal agile-eye, proposed in a previous investigation [3]. The link lengths $\lambda, \gamma$, and $\xi$ decreased from $90^{\circ}$ in [3] to $64^{\circ}$, $69^{\circ}, 35^{\circ}$, respectively, in the current study, which indicates a considerable size reduction. Also, the maximum condition number reduced from 2.71 to 1.31 , which corresponds to improvement in isotropy.

Table 2 reports the results of the kinematic assessment tests in terms of the maximum and rms errors for the RCM position, and the tracking accuracy for the conical and inplane tests. Each reported value includes the average and standard deviation for each parameter throughout 10 trials. The maximum and rms errors of the RCM displacement were below $0.83 \mathrm{~mm} \pm 0.05 \mathrm{~mm}$ and $0.51 \mathrm{~mm} \pm 0.04 \mathrm{~mm}$, and those of the tracking less that $0.52^{\circ} \pm 0.02^{\circ}$ and $0.27^{\circ} \pm 0.01^{\circ}$, respectively. The worst case for conical tests occurred at $\phi=25^{\circ}$, which is the boundary of the target workspace. For in-plane tests, except for $\theta=90^{\circ}$, the errors were in the same 
(a)

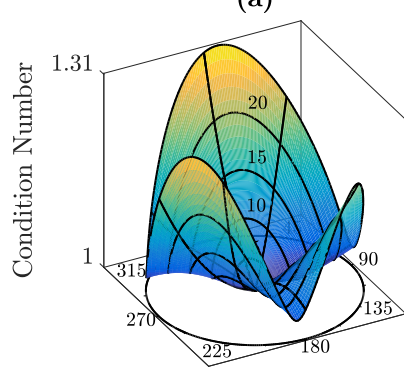

(b)

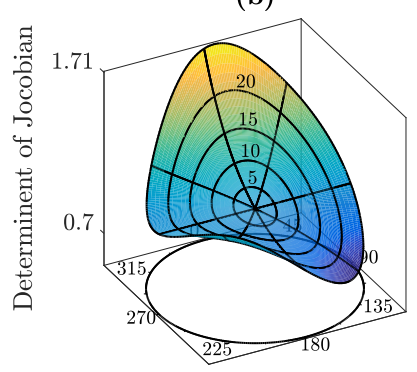

Fig. 8. (a) Condition number and (b) determinant of Jacobian for the optimized design of the mechanism. Numbers on the bottom plane and the graph indicate the angles $\theta$ and $\phi$ of the spherical coordinate, respectively.

TABLE I

LINK LENGTHS AND PERFORMANCE MEASURES OF THE CURRENT AND PREVIOUSLY PROPOSED AGILE-EYE MECHANISMS

\begin{tabular}{|l|c|c|}
\hline & $\begin{array}{c}\text { Non-symmetric } \\
\text { (current) design }\end{array}$ & $\begin{array}{c}\text { Orthogonal design } \\
\text { (proposed by [3]) }\end{array}$ \\
\hline \multirow{4}{*}{ Link } & $\alpha=32^{\circ}$ & $\alpha=45^{\circ}$ \\
lengths & $\beta=0^{\circ}$ & $\beta=-30^{\circ}$ \\
& $\lambda=64^{\circ}$ & $\lambda=90^{\circ}$ \\
& $\gamma=69^{\circ}$ & $\gamma=90^{\circ}$ \\
& $\xi=35^{\circ}$ & $\xi=90^{\circ}$ \\
\hline Performance & $C N<1.31$ & $C N<2.71$ \\
& $0.7<J<1.71$ & $0.47<J<1.06$ \\
\hline
\end{tabular}

range, but worst for $\theta=0^{\circ}$. Fig. 9 shows the tracking error vs time for conical and in-plane tests that involved the maximum errors.

Table 3 reports the results of the load-carrying capability tests in terms of the maximum and rms of the agile-eye's motor torques, for two conical trajectories and three external moment conditions. To investigate the load-carrying capability and isotropy more closely, and also assess the relationship between external loads and motor torques, the net effect of the external moment was extracted from the actual motor torque. This was accomplished by subtracting the motor torques recorded for tests with external moment from those of free running tests. The results are illustrated in Fig. 10, which compares the net motor torques, obtained as $\boldsymbol{\tau}_{\text {Exp }}=\mathbf{J}^{T} \boldsymbol{\tau}_{s}$, with the actual torque for $\phi=15^{\circ}$ conical trajectory.

To study the effect of the external load on the accuracy

(a)

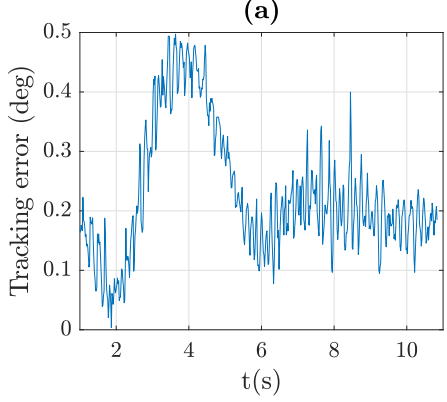

(b)

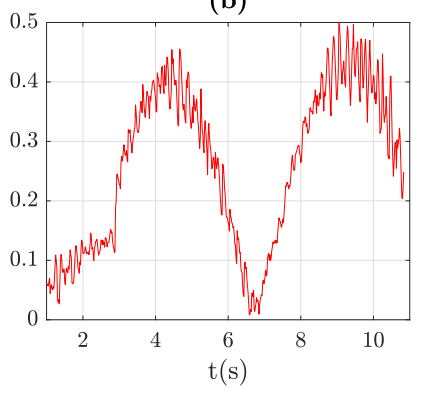

Fig. 9. Tracking error vs time for (a) $\phi=25^{\circ}$ conical trajectory and (b) $\theta=0^{\circ}$ in-plane trajectory
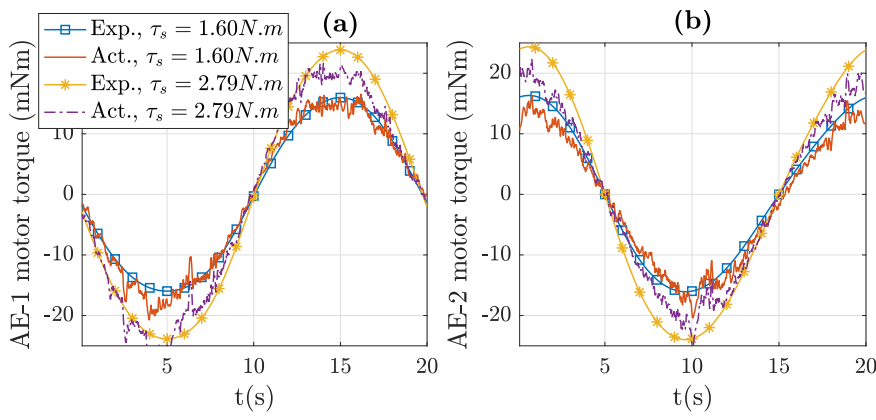

Fig. 10. Actual and net torques of (a) first and (b) second agile-eye motors for $\phi=15^{\circ}$ conical trajectory, under two different external moments

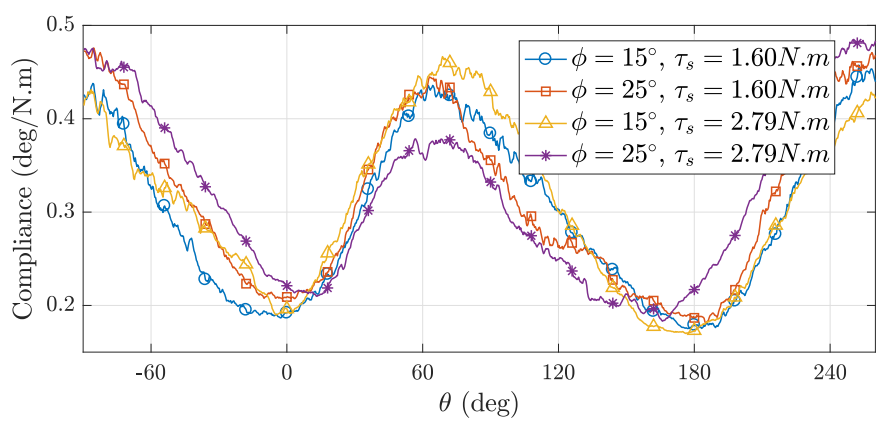

Fig. 11. Compliance of agile-eye mechanism vs azimuth angle

of the tool manipulator, the mechanism's compliance was investigated. This was accomplished by calculating the orientation change, recorded from tests with similar trajectories but different external moments. The results are shown in Fig. 11. The compliance of the first and second kinematic chains can be roughly estimated as $0.19 \mathrm{deg} / \mathrm{N} . \mathrm{m}$ and $0.45 \mathrm{deg} / \mathrm{N} . \mathrm{m}$, respectively.

Finally, Fig. 12 depicts the wrist angle, i.e. the angle between the tool-tip and the tool shaft, denoted by $\psi$, for conical trajectories $\phi=5^{\circ}, \phi=15^{\circ}$, and $\phi=25^{\circ}$, versus azimuth angle $\theta$. The gray grid circles in the figure indicate $\psi=15^{\circ}, 45^{\circ}$ and $75^{\circ}$. The dashed yellow curve corresponds to $\phi=25^{\circ}$ and shows that the intended workspace for the instrument wrist, i.e. $\psi=75^{\circ}$ is achieved. This figure also shows that the ratio between the handle inclination, $\phi$, and the wrist angle, $\psi$, is not constant and changes with $\theta$ as well as $\phi$.

\section{DISCUSSION AND CONCLUSION}

The usage of the handheld wristed laparascopic instruments in robotic surgery has drawn attention in the recent years. This paper described the design, optimization, prototyping and experimental study of an effective robotic wrist manipulator for this purpose. This manipulator is based on the novel nonsymmetric 5R-SPM structure, which enjoys orthogonal links in one chain and links with arbitrary lengths in the other. The RCM mechanism with a large workspace makes the manipulator capable of activating the spherical joint, between the handle and the tool, of a large variety of commercial handheld wristed laparoscopic instruments, e.g. RealHand (Novare 
TABLE II

RCM ERROR AND TRACKING ERROR FOR CONICAL AND IN-PLANE TESTS, WITH NO EXTERNAL MOMENT

\begin{tabular}{|cc|c|c|c|c|c|c|c|}
\hline & & $\phi=5$ & $\phi=15$ & $\phi=25$ & $\theta=0$ & $\theta=45$ & $\theta=90$ & $\theta=135$ \\
\hline RCM Err. & $\max$ & $0.25 \pm 0.04$ & $0.58 \pm 0.05$ & $0.72 \pm 0.03$ & $0.48 \pm 0.06$ & $0.17 \pm 0.04$ & $0.55 \pm 0.05$ & $0.83 \pm 0.05$ \\
$(\mathrm{~mm})$ & $\mathrm{rms}$ & $0.11 \pm 0.02$ & $0.30 \pm 0.03$ & $0.43 \pm 0.02$ & $0.31 \pm 0.04$ & $0.05 \pm 0.02$ & $0.37 \pm 0.03$ & $0.51 \pm 0.04$ \\
\hline Tracking Err. & $\max$ & $0.15 \pm 0.01$ & $0.35 \pm 0.01$ & $0.50 \pm 0.02$ & $0.51 \pm 0.02$ & $0.52 \pm 0.02$ & $0.25 \pm 0.01$ & $0.43 \pm 0.02$ \\
(deg) & $\mathrm{rms}$ & $0.06 \pm 0.01$ & $0.17 \pm 0.01$ & $0.26 \pm 0.02$ & $0.27 \pm 0.01$ & $0.17 \pm 0.01$ & $0.11 \pm 0.01$ & $0.26 \pm 0.02$ \\
\hline
\end{tabular}

TABLE III

ACTUAL TORQUES FOR DIFFERENT EXTERNAL MOMENTS

\begin{tabular}{|l|c|c|c|c|c|c|}
\hline & \multicolumn{2}{|c|}{$\tau_{s}=0.0 N . m$} & \multicolumn{2}{c|}{$\tau_{s}=1.60 N . m$} & \multicolumn{2}{c|}{$\tau_{s}=2.79 N . m$} \\
\cline { 2 - 7 } & $\phi=15$ & $\phi=25$ & $\phi=15$ & $\phi=25$ & $\phi=15$ & $\phi=25$ \\
\hline Motor 1 max torque $(\mathrm{mNm})$ & 21 & 22 & 31 & 28 & 39 & 38 \\
\hline Motor 2 max torque $(\mathrm{mNm})$ & 20 & 22 & 29 & 26 & 34 & 32 \\
\hline Motor 1 rms torque (mNm) & 12 & 13 & 16 & 16 & 20 & 21 \\
\hline Motor 2 rms torque (mNm) & 12 & 13 & 14 & 14 & 17 & 17 \\
\hline
\end{tabular}

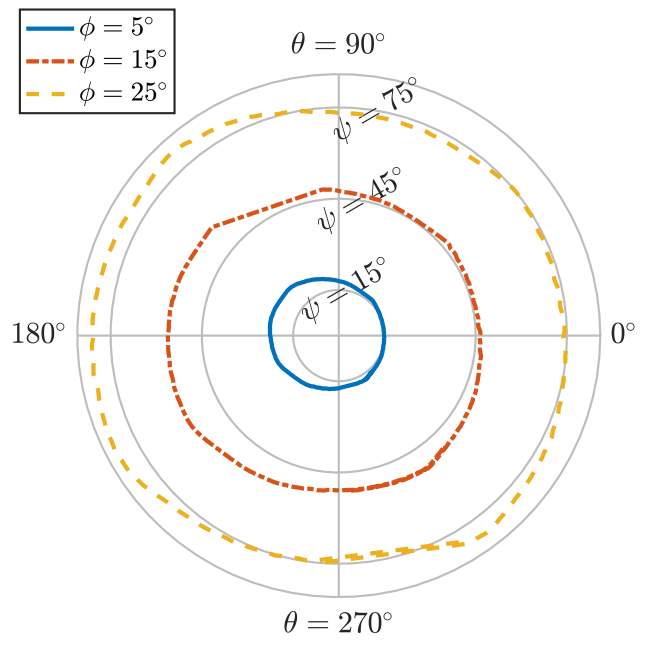

Fig. 12. Wrist orientation with respect to tool shaft for three conical tests $\phi=$ $5^{\circ}, \phi=15^{\circ}$, and $\phi=25^{\circ}$. The circular grid shows $\psi=15^{\circ}, 45^{\circ}$, and $75^{\circ}$.

Surgical Systems, Inc.) [5], Autonomy Laparo-Angle Articulating Instruments (Cambridge Endoscopic Devices, Inc.) [6], and SILS Hand Instruments (Medtronic-Covidien) [7]. More specifically, the non-symmetric agile-eye mechanism, with smaller links in the second kinematic chain, enables avoiding collisions, reducing the size of the mechanism, and increasing its stiffness.

The optimization results (Fig. 8 and Table 1) indicate that the proposed mechanism can provide a great manipulabilty and a high isotropy by appropriate selection of the link lengths and the location on the robot base. In particular, in comparison with the orthogonal agile-eye mechanism investigated in [3], the condition number decreased by more than $50 \%$ and the minimum value for the determinant of the Jacobian increased by about $45 \%$ (Table 1). Moreover, the size and weight of the mechanism were reduced substantially due to the smaller lengths of the links $\lambda, \gamma$, and $\xi$. Neverthelss, these changes were accompanied with $\alpha=\lambda / 2$ and $\beta=0$ which indicates symmetric and horizontal placement of actuators, in spite of the nonsymetry of the mechanism.

The results of the kinematic assessment tests (Table 2) indicate a nearly constant RCM point, with sub-millimeter displacements. Also, the tracking accuracy was reasonably high, with rms error in the range of $0.06 \mathrm{~mm}$ to $0.27 \mathrm{~mm}$. The maximum tracking error for conical tests occurred at $\phi=25^{\circ}$, which was the boundary of the target workspace. This observation is not unexpected since, in such large maneuvers, the errors associated with the fabrication tolerances, joint flexibilities, and end-effector's weight are highlighted. For in-plane tests, the largest tracking errors occurred at $\theta=0^{\circ}$. The tracking error of these two worst cases are shown against time in Fig. 9. The high frequency noise of the data is due to the errors associated with the NDI tracker, which is not significant compared with the observed tracking error. According to Fig. 9(b), the tracking error peaks around $\mathrm{t}=5 \mathrm{~s}$ and $\mathrm{t}=10 \mathrm{~s}$, both corresponded to the boundary of in-plane trajectory, i.e. $\phi=25^{\circ}$. Again, this behavior can be justified by the fact that the errors are magnified when the end-effector is mostly inclined. As the figure shows, the tracking error became almost zero near $t=7 \mathrm{~s}$, when the end-effector was close to the vertical position.

In general, the results of the kinematic assessment tests (Table 2) indicate that although the accuracy of the robot was reasonably acceptable, its repeatability was substantially high. This result suggests that the backlash and mechanical looseness were negligible in the fabricated prototype and most of the errors came from the geometrical insufficiencies such as dimensional and assembly errors. Such inaccuracies can be considerably decreased by performing a simple kinematic calibration on the mechanism in future.

The results of the load-carrying capability tests (Table 3 ) indicate that the designed robot, based on a non-symmetric agile-eye mechanism, enjoyed a great isotropy. In all tests, the maximum and rms torques of the two motors were very close, suggesting that a highly isotropic design was achieved and the actuators were selected appropriately. These suggestions are confirmed by Fig. 10 which shows that the range of the required motor torques for realizing the predefined trajectories were nearly the same. Moreover, this figure indicates that the net motor torques, obtained as $\boldsymbol{\tau}_{\text {Exp }}=\mathbf{J}^{T} \boldsymbol{\tau}_{s}$, and the actual motor torques from external moments match closely. 
The results of the compliance analysis (Fig. 11) indicate similar compliance behavior in different test conditions. In general, the agile-eye mechanism was stiffest at $\theta=0^{\circ}$ and $\theta=180^{\circ}$, and softest near to $\theta=60^{\circ}$. These behaviors might be explained by considering the geometry of the mechanism chains at these configurations. In the first two configurations, the axis of the external moment became aligned with the axis of the first motor, hence, the external moment was transferred to the base mainly through the first kinematic chain. Referring to Fig. 2, this kinematic chain contains a rigid Y shaped link and a double supported joint (joint 5), providing a very high stiffness. On the other hand, considering the fact that the angle between the axis of the agile-eye motors is $64^{\circ}$, the external moment and the second motor's axis became aligned near $\theta=$ $60^{\circ}$. Hence, the external moment was mainly withstood by the second kinematic chain at this configuration. This chain is much more yielding to external loads than the first kinematic chain, due to its higher number of links and joints, as well as its weaker joints. Based on this observation, the stiffness of the agile-eye's second kinematic chain needs more attention. To address this issue, we suggest a more detailed structural analysis and performing an optimization procedure that also considers the stiffness of the chains in the cost function.

In the end, the results of the instrument test (Fig. 12) show that the ratio between the handle inclination, $\phi$, and the wrist angle, $\psi$, is not constant and depends on the orientation of the handle, i.e. $\theta$ and $\phi$. The variation of this ration was magnified for experiments with smaller handle inclination, $\phi$. This nonlinear behavior is thought to be associated with the inherent backlash and deflection of the actuation cables attaching the handle and the tool-tip, as well as the changing stiffness of the snake-like wrist. Therefore, to achieve a high tracking accuracy at the tool-tip, a thorough investigation on kinematic modeling and calibration of the wristed instrument is required.

\section{ACKNOWLEDGMENT}

The authors wish to thank Sina Robotic and Medical Innovators Co. and also Parsiss Surgical Navigation Co. for facilitating the experiments.

\section{REFERENCES}

[1] P. Van, J. Hauspy, L. Verkinderen, B. Trinh, L. Van, and L. Dirix, "Do Costs of Robotic Surgery Matter?" in Adv. Gynecol. Endosc., 2012.

[2] R. Prewitt, V. Bochkarev, C. L. McBride, S. Kinney, and D. Oleynikov, "The patterns and costs of the da Vinci robotic surgery system in a large academic institution," J. Robot. Surg., vol. 2, no. 1, pp. 17-20, 2008.

[3] S. Hanifeh, A. Alamdar, F. Farahmand, and A. Mirbagheri, "An Adapting Mechanism to Manipulate Manual Wristed Laparoscopic Instruments by a Robotic Surgery System," in 4th Int. Conf. Control. Dyn. Syst. Robot., no. 107, 2017, pp. 1-8.

[4] F. Farahmand, A. Mirbagheri, S. Behzadipour, A. Alamdar, S. Hanifeh, S. Khadem, and M. Moradi, "Adapting manual laparoscopic surgical instruments for robotic telesurgery applications," 2016.

[5] C. W. Jeong, S. H. Kim, H. T. Kim, S. J. Jeong, S. K. Hong, S.-S. Byun, and S. E. Lee, "Insufficient Joint Forces of First-Generation Articulating Instruments for Laparoendoscopic Single-Site Surgery," Surg. Innov., vol. 20, no. 5, pp. 466-470, oct 2013.

[6] S. K. Kim, J. H. Lee, J. R. Lee, C. S. Suh, and S. H. Kim, "Laparoendoscopic Single-site Myomectomy Versus Conventional Laparoscopic Myomectomy: A Comparison of Surgical Outcomes," J. Minim. Invasive Gynecol., vol. 21, no. 5, pp. 775-781, 2014.
[7] S. L., B. F., N. F., T. F.R., and C. L., "Laparoendoscopic single-site partial nephrectomy without ischemia for very small, exophytic renal masses: Surgical details and functional outcomes," Eur. Urol., vol. 63, no. 4, pp. 759-765, 2013.

[8] F. Cianchi, F. Staderini, and B. Badii, "Single-incision laparoscopic colectomy: A new era in the treatment of colorectal cancer?" Colorectal Cancer: Surgery, Diagnostics and Treatment, p. 125, 2014.

[9] N. Yoshiki, "Single-incision laparoscopic myomectomy: A review of the literature and available evidence," Gynecology and Minimally Invasive Therapy, vol. 5, no. 2, pp. 54-63, 2016.

[10] P. L. Anderson, R. A. Lathrop, and R. J. Webster, "Robot-like dexterity without computers and motors: a review of hand-held laparoscopic instruments with wrist-like tip articulation," Expert Rev. Med. Devices, vol. 13, no. 7, pp. 661-672, 2016.

[11] T. A. Ponsky, "Single Port Laparoscopic Cholecystectomy in Adults and Children: Tools and Techniques," J. Am. Coll. Surg., vol. 209, no. 5, pp. e1-e6, 2009.

[12] Sánchez-Margallo, F. M., J. A. Sánchez-Margallo, and A. Szold, "Handheld Devices for Laparoscopic Surgery," in New Horizons Laparosc. Surg., 2018, vol. i, p. 13.

[13] C.-H. Kuo, J. S. Dai, and P. Dasgupta, "Kinematic design considerations for minimally invasive surgical robots: an overview," Int. J. Med. Robot. Comput. Assist. Surg., vol. 8, no. 2, pp. 127-145, jun 2012.

[14] Y. D. Patel and P. M. George, "Parallel Manipulators Applications-A Survey," Mod. Mech. Eng., vol. 02, no. 03, pp. 57-64, 2012.

[15] Z. Tao and Q. An, "Interference analysis and workspace optimization of 3-RRR spherical parallel mechanism," Mech. Mach. Theory, vol. 69, pp. 62-72, 2013.

[16] J. Yu, K. Wu, G. Zong, and X. Kong, "A Comparative Study on Motion Characteristics of Three Two-Degree-of-Freedom Pointing Mechanisms," J. Mech. Robot., vol. 8, no. 2, p. 021027, 2016.

[17] G. Wu, S. Caro, and J. Wang, "Design and transmission analysis of an asymmetrical spherical parallel manipulator," Mech. Mach. Theory, vol. 94, pp. 119-131, dec 2015.

[18] B. M. Schena, "Center robotic arm with five-bar spherical linkage for endoscopic camera," 2012.

[19] M. Dehghani, M. M. Moghadam, and P. Torabi, "Analysis, optimization and prototyping of a parallel RCM mechanism of a surgical robot for craniotomy surgery," Ind. Rob., vol. 45, no. 1, pp. 78-88, 2018.

[20] A. Alamdar, S. Hanife, F. Farahmand, S. Behzadipour, and A. Mirbagheri, "A minimally invasive robotic surgery approach to perform totally endoscopic coronary artery bypass on beating hearts," Med. Hypotheses, vol. 124, pp. 76-83, mar 2019.

[21] T. Essomba, M. A. Laribi, S. Zeghloul, and G. Poisson, "Optimal synthesis of a spherical parallel mechanism for medical application," Robotica, vol. 34, no. 3, pp. 671-686, 2016.

[22] T. Essomba and L. Nguyen Vu, "Kinematic analysis of a new five-bar spherical decoupled mechanism with two-degrees of freedom remote center of motion," Mech. Mach. Theory, vol. 119, pp. 184-197, 2018.

[23] C. M. Gosselin and F. Caron, "Two degree-of-freedom spherical orienting device," 1999.

[24] A. Alamdar, F. Farahmand, S. Behzadipour, and A. Mirbagheri, "A geometrical approach for configuration and singularity analysis of a new non-symmetric 2 dof $5 \mathrm{r}$ spherical parallel manipulator," Mechanism and Machine Theory, vol. 147, p. 103747, 2020. 